\title{
„Choice and voice“ in Schulen mit personalisierten Lernkonzepten aus Sicht der Schülerinnen und Schüler
}

\author{
Christine Mötteli (iD · Urs Grob • Christine Pauli • Kurt Reusser • \\ Rita Stebler
}

Eingegangen: 29. September 2020 / Überarbeitet: 7. Mai 2021 / Angenommen: 4. Juni 2021 / Online publiziert: 1. Juli 2021

(C) Der/die Autor(en) 2021

Zusammenfassung Choice and voice ist eine zentrale Forderung im Kontext personalisierter Lernkonzepte und steht für den Anspruch den Schüler*innen Wahl- und Mitbestimmungsmöglichkeiten einzuräumen. Unabhängig der neuen Rhetorik haben verschiedene innovative Schulen langjährige Praxiserfahrung mit Wahl- und Mitbestimmungsmöglichkeiten, wobei wenig über deren Wahrnehmung aus Sicht der Schüler*innen bekannt ist. Der vorliegende Beitrag untersucht, wie Schüler*innen der Sekundarstufe I choice and voice in Schulen mit personalisierten Lernkonzepten wahrnehmen.

Die Ergebnisse zeigen, dass die Schüler*innen regelmäßig Wahlfreiheit wahrnehmen, insbesondere was lernorganisatorische Fragen betrifft. Die Möglichkeit, Inhalte und Ziele mitzubestimmen, nehmen sie als weniger ausgeprägt wahr. Der Personalisierungsgrad als Maß dafür, wie stark sich eine Schule aus Sicht der Lehrpersonen an personalisierten Lernkonzepten orientiert, erweist sich in einem Mehrebenen-Strukturgleichungsmodell als relevanter Prädiktor zur Vorhersage der Wahrnehmung der Schüler*innen in Bezug auf Wahl- und Mitbestimmungsmöglichkeiten. Des Weiteren zeigt sich, dass häufiger wahrgenommene Wahl- und Mitbestimmungsmöglichkeiten mit einer stärker positiv wahrgenommenen Unterstützung der Lehrperson einhergehen.

Der Artikel leistet einen Beitrag zur Klärung des vielschichtigen Personalisierungsbegriffes, indem er zeigt, dass choice and voice nicht nur aus theoretischer, sondern auch aus empirischer Sicht eine wichtige Dimension personalisierter Lernkonzepte darstellt.

Christine Mötteli $(\varangle) \cdot$ Urs Grob $\cdot$ Kurt Reusser $\cdot$ Rita Stebler

Institut für Erziehungswissenschaft, Universität Zürich, Zürich, Schweiz

E-Mail: christine.moetteli@uzh.ch

Christine Pauli

Zentrum für Lehrerinnen- und Lehrerbildung, Universität Freiburg, Freiburg, Schweiz 
Schlüsselwörter Personalisiertes Lernen · Wahlfreiheit · Mitbestimmung · Erweiterte Autonomie

\section{Choice and voice from the students' point of view in schools with personalised learning environments}

Abstract Choice and voice is a central requirement in the context of personalised learning concepts. Despite contemporary rhetoric, a variety of innovative schools have gained many years of practical experience with choice and voice, but a lot less is known about the perceptions of this approach from the point of view of students. This article examines how students at secondary level I perceive choice and voice in schools with personalised learning environments.

Findings indicate that students regularly exercise their freedom of choice, particularly with regard to questions concerning the organisation of learning. The enthusiasm of students for participation in determining content and goals is less pronounced. The degree of personalisation, which indicates how strongly a school is motivated by personalised learning concepts from the point of view of teachers, proves to be a relevant predictor in the multilevel structural equation model of the awareness of students regarding choice and voice options. Correlations in multilevel structural equation modelling also illustrate that awareness of choice and voice options is frequently accompanied by a positive perception and support of the teacher.

This article contributes to the explanation of the complex term of personalisation by demonstrating that, in addition to its theoretical significance, choice and voice also represents an important dimension of personalised learning concepts from an empirical point of view.

Keywords Personalised/Personalized Learning - Choice and voice · Participation · Autonomy support

\section{Einleitung}

Die Auseinandersetzung mit Wahl- und Mitbestimmungsmöglichkeiten hat in der Schulpädagogik eine lange Tradition. Bereits im letzten Jahrhundert wurde die Thematik im Kontext der Reformpädagogik international diskutiert (Oelkers 2010). In der neueren deutschsprachigen Literatur werden erweiterte Autonomiespielräume im Rahmen von Formen des offenen Unterrichts und des selbstregulierten Lernens gefordert, um heterogenen Lerngruppen einen niveauangepassten, selbstbestimmten, motivierenden und lernkompetenzfördernden Unterricht zu ermöglichen (Bohl und Kucharz 2010; Götz und Nett 2017). Seit rund 15 Jahren wird die Diskussion darüber insbesondere im angloamerikanischen Raum durch den Begriff des personalisierten Lernens erweitert, wobei unter dem Slogan choice and voice Wahlmöglichkeiten und die aktive Mitgestaltung von Schule und Unterricht seitens der Lernenden gefordert wird (Bray und McClaskey 2015; Miliband 2006). In der Deutschschweiz orientieren sich verschiedene innovative Schulen ebenfalls an personalisierten Lernkonzepten, indem sie ihren Schüler*innen einen individualisierenden, selbstorganisierten sowie 
fachlich und überfachlich kompetenzfördernden Unterricht zu ermöglichen suchen. Obwohl manche Schulen bereits langjährige Erfahrung mit vielfältigen autonomiefördernden Lernumgebungen aufweisen, mangelt es immer noch an wissenschaftlichen Untersuchungen zu Dimensionen und Umsetzungsformen der Autonomiegewährung. Diese Forschungslücke soll mit dem vorliegenden Beitrag bearbeitet werden, indem untersucht wird, welche Grade an Wahl- und Mitbestimmungsmöglichkeiten Schüler*innen in Schulen mit personalisierten Lernkonzepten wahrnehmen. Damit soll zur Klärung der Frage beigetragen werden, inwiefern es sich bei Wahlund Mitbestimmungsmöglichkeiten um empirisch bedeutsame Konzeptdimensionen personalisierten Lernens handelt. Die ausgewerteten Daten stammen aus der perLenStudie (personalisierte Lernkonzepte in heterogenen Lerngruppen) (Stebler et al. 2018), in der Lehr-Lernkulturen untersucht werden, die dem Sammelbegriff personalisierter Lernkonzepte zugeordnet werden können. Als theoretischer Rahmen werden das Konzept des personalisierten Lernens und die damit häufig verbundenen Begriffe choice and voice in lernpsychologischer Perspektive eingeführt. Zur Kontextverortung werden die Bedeutung von Wahl- und Mitbestimmungsmöglichkeiten aus bildungspolitischer Sicht sowie deren Verbreitung und Wirkungsweisen aus empirischer Sicht dargelegt. Im empirischen Teil steht die Wahrnehmung von choice and voice aus Sicht der Schüler*innen im Zentrum. Die Auswertungen sollen zu einem tieferen Verständnis der praktischen Umsetzung personalisierter Lernkonzepte anhand von Daten aus der Deutschschweiz beitragen.

\section{Theoretischer Rahmen}

\subsection{Personalisiertes Lernen - Ein internationales Bildungsschlagwort}

Unter dem Stichwort personalisiertes Lernen werden international Lernkonzepte diskutiert, welche sich an den individuellen Voraussetzungen und Bedürfnissen der Lernenden orientieren und eine aktive Beteiligung der Schüler*innen mittels choice and voice ermöglichen (Bray und McClaskey 2015; DfES 2004a; Keamy et al. 2007; OECD o.J.; Schratz und Westfall-Greiter 2010; U.S. Department of Education 2017a). Obwohl die OECD (2006) bereits vor rund 15 Jahren Personalisierung als Bildungskonzept der Zukunft diskutierte, fehlt bis anhin ein einheitliches Verständnis dieses vielschichtigen Begriffs (Keamy et al. 2007; Prain et al. 2013; Stebler et al. 2018; U.S. Department of Education 2017b). Nebst unterschiedlichen Akzentuierungen verschiedener Facetten personalisierten Lernens, unterscheiden sich auch die Vorstellungen zur praktischen Umsetzung, welche von einer Unterrichtsentwicklung innerhalb bestehender Strukturen bis hin zu einem kompletten Neudenken von Unterricht und den Rollen der Beteiligten reichen (Bray und McClaskey 2015; DfES 2004a; Lee et al. 2018; Sebba et al. 2007; U.S. Department of Education 2017b). Für die perLen-Studie haben Stebler et al. (2018, 2021) ausgehend vom internationalen Diskurs (u.a. Bray und McClaskey 2015; DfES 2004a; Hoz 1986; Miliband 2006; Müller 2014; Murphy et al. 2016; Schratz und Westfall-Greiter 2010; U.S. Department of Education 2017a) fünf Dimensionen personalisierten Lernens herausgearbeitet, welche in unterschiedlichen Akzentuierungen als handlungsleitende 
Grundsätze bzw. Orientierungen dienen: 1) Unterrichtsangebote an die personalen Bildungs- und Lernvoraussetzungen von Lernenden und Lerngruppen anpassen; 2) personale und soziale Kompetenzen aufbauen und dadurch Schüler*innen in ihrer Persönlichkeit ganzheitlich fördern; 3) selbstgesteuertes Lernen auf eigenen Wegen ermöglichen; 4) als Lernende kompetenzorientiertes Lernen zur persönlichen Sache machen; 5) als Lehrperson und als Lerngemeinschaft bildend und unterstützend wirken.

Obgleich der Begriff des personalisierten Lernens in der deutschsprachigen Literatur bislang nicht stark verbreitet ist (z. B. Agostini et al. 2018; Holmes et al. 2018; Stebler et al. 2018), zeigt sich eine nahe Verwandtschaft zu den im deutschsprachigen Raum intensiv diskutierten Konzepten der Individualisierung, Differenzierung, des adaptiven Unterrichts, des offenen Unterrichts oder des selbstregulierten Lernens (Stebler et al. 2021). Während beim differenzierenden und individualisierenden Lernen sowie beim adaptiven Unterricht die Frage nach der Passung von Lernangebot und Lernbedürfnis im Vordergrund steht, fokussieren der offene Unterricht und das selbstregulierte Lernen darüber hinaus Fragen nach der Steuerung und Verantwortungsübernahme im Lernprozess. Beide Aspekte sind Kernelemente personalisierter Lernkonzepte und werden in der Literatur unter dem Slogan choice and voice diskutiert.

\subsection{Choice and voice in personalisierten Lernkonzepten}

An einer OECD-Konferenz zum Begriff des personalisierten Lernens von 2004 plädierte der damalige britische Staatsminister für Schulen, David Miliband, für deutlich mehr choice and voice in allen Belangen von Schule und beeinflusste damit den Personalisierungsdiskurs nachhaltig (Miliband 2006). Der Slogan choice and voice wurde von verschiedenen Autorinnen und Autoren aufgegriffen, jedoch meist ohne Milibands wirtschaftlich orientierte Interpretation zu übernehmen. Milibands Verständnis fußte auf einem Personalisierungsbegriff, der seine Wurzeln in der Marketing-Theorie hat und bei dem die aktive Mitgestaltung von Prozessen und Produkten durch Kundinnen und Kunden im Vordergrund steht (Hartley 2008). Durch choice and voice sollen Schüler*innen sowie deren Eltern auf allen Ebenen des Schulsystems nicht nur Wahlmöglichkeiten (choice) erhalten, sondern die damit verbundenen Wahloptionen mitgestalten können, indem sie ihre Ideen und Wünsche zur Weiterentwicklung des Bildungsangebotes einbringen können (voice) (Miliband 2006; OECD o.J.). Das britische Bildungsdepartement (Department for Education and Skills, [DfES]) beschreibt die praktische Umsetzung von choice and voice im Rahmen eines lehrpersonengesteuerten und vom traditionellen Unterricht nur marginal abweichenden Unterrichts. Danach diagnostizieren die Lehrpersonen Kompetenzstand und präferierte Lernmethoden der Schüler*innen, beobachten, welche Themen die Lernenden besonders interessant finden und gestalten entsprechende Unterrichtsarrangements. Innerhalb dieser vorbereiteten Settings erhalten die Lernenden sodann bestimmte Wahlmöglichkeiten (choice). Mit zunehmendem Alter der Lernenden erweitern sich die inhaltlichen Wahlmöglichkeiten innerhalb des staatlichen Curriculums (DfES 2004a, b). Damit die Lehrpersonen maßgeschneiderte Angebote planen können, müssen die Schüler*innen ihre Wünsche und Bedürfnisse 
einbringen können (voice). Inwiefern die Lernenden am Wahlangebot aktiv mitwirken können, wird nicht explizit erläutert. Choice and voice bedeutet in diesem Sinne primär, dass die Lehrpersonen im Unterricht Wahlmöglichkeiten anbieten und die Lernenden anhören, damit deren Wünsche und Bedürfnisse in der Unterrichtsplanung berücksichtigt werden können. In der unterrichtsnahen, stärker an Konzepten der Differenzierung und Individualisierung des Lernens ausgerichteten Publikation „Make Learning Personal“ von Bray und McClaskey (2015) entfällt die klassische Planungsfunktion der Lehrperson zugunsten einer strikten Orientierung des Unterrichts an den Lernenden, wodurch die Idee des sich aktiven Einbringens mittels choice and voice eine nochmals erweiterte Akzentuierung erhält. Die Schüler*innen übernehmen bei der Wahl der Inhalte, der Spezifizierung der Lernziele und wie sie diese erreichen wollen (voice), eine deutliche Mitverantwortung. Auch bezüglich der Rechenschaftslegung haben sie Wahlmöglichkeiten (choice), wie sie ihre Kompetenzen zeigen wollen. Dadurch, dass die Gestaltung und Verantwortung für das Lernen im Unterricht - choice and voice - in deutlicher Akzentsetzung an die Lernenden übertragen wird, grenzen die Autorinnen personalisiertes Lernen von den Lehrpersonen verantworteten Formen der Differenzierung und Individualisierung ab (Bray und McClaskey 2015).

Inwiefern die Lernenden durch choice and voice an Entscheidungen partizipieren können, hängt, wie die beiden Beispiele zeigen, vom Ausmaß der in die Mitsprache und Mitverantwortung der Lernenden einbezogenen Dimensionen ab. Im Artikel wird unter choice and voice im Sinne des DfES ein Wahlangebot verstanden (choice), welches von den Schüler*innen mitgestaltet werden kann (voice) und sich sowohl auf Inhalte als auch auf den Lernprozess bezieht. Während choice im vorliegenden Artikel mit Wahlmöglichkeiten übersetzt wird, übersetzen wir voice - da es hier primär darum geht, dass sich die Lernenden einbringen können (,eine Stimme geben“) - mit Mitbestimmung. Choice and voice verortet sich im Rahmen personalisierten Lernens somit in den von Stebler et al. (2018, 2021) herausgearbeiteten Dimensionen eins (angepasstes Bildungsangebot), drei (selbstgesteuertes Lernen) und vier (Lernen zur persönlichen Sache machen). Welche Bedeutung dem Begriffspaar in der Praxis sowie in einer theoretischen und empirischen Perspektive zukommt, wird in den folgenden Kapiteln dargelegt.

\subsection{Choice and voice in Deutschschweizer Schulen}

In der Deutschschweiz ist ein Recht auf Mitverantwortung und Mitsprache von Schüler*innen aus pädagogischer und auch rechtlicher Sicht weitgehend unbestritten und in einigen Kantonen explizit gesetzlich festgeschrieben (z.B. § 50 Volksschulgesetz Kanton Zürich vom 7. Februar 2005 [VSG; LS 412.100]). Gemäß Lehrplan 21 ist die Kompetenz, zunehmend selbstständig zu lernen, ein bedeutsames Ziel der Volksschule (Bildungsdirektion Kanton Zürich 2017), dessen Förderung ein Unterrichtsangebot bedingt, in welchem die Lernenden selbst Entscheidungen in niveauangepassten Freiräumen fällen können.

Im Hinblick auf die Praxisumsetzung haben Wahl- und Mitbestimmungsmöglichkeiten in der Deutschschweiz insbesondere seit den 1990er-Jahren durch die Verbreitung der „Erweiterten Lehr- und Lernformen“ (ELF) Eingang in die Klassenzimmer 
gefunden. Bei den ELF handelt es sich um eine von der Praxis ausgehende und von dieser getragene Reformbewegung in der Deutschschweiz, welche ,auf eine stärkere Individualisierung und Förderung des selbstverantwortlichen und selbstgesteuerten Lernens durch vermehrten Einbezug offener Unterrichtsformen zielte" (Pauli et al. 2010, S. 314). In Abgrenzung zum vor allem in Deutschland diskutierten Konzept des offenen Unterrichts wurden ELF nicht als Ersatz, sondern als Erweiterung traditioneller Unterrichtsformen gedacht (Pauli et al. 2010). Vor rund 20 Jahren gaben $92 \%$ der befragten Deutschschweizer Lehrpersonen an, zumindest ab und zu ELF im Mathematikunterricht einzusetzen (Pauli et al. 2003). Ein Vergleich von Lehrpersonen, welche häufig oder fast immer ELF einsetzen (ELF-Gruppe) und Lehrpersonen, welche nur selten oder sporadisch ELF einsetzen (Nicht-ELF-Gruppe) zeigte, dass Lernende der ELF-Gruppe vermehrt über Freiräume und Mitsprachemöglichkeiten berichten (Pauli et al. 2003). Eine aktuellere Studie, welche die unterrichtsbezogenen Partizipationsmöglichkeiten in Klassen der Primar- und Sekundarstufe I untersuchte, zeichnet ein weniger optimistisches Bild. Nach Müller-Kuhn et al. (2020) berichten sowohl die rund 500 befragten Schüler*innen als auch deren Lehrpersonen von wenig Wahl- und Mitbestimmungsmöglichkeiten im Unterricht, wobei die Möglichkeiten von den Lehrpersonen signifikant höher eingeschätzt werden.

\subsection{Choice and voice aus theoretischer und empirischer Sicht}

Im deutschsprachigen Raum hat sich das Angebot-Nutzungs-Modell (Helmke 2009; Reusser und Pauli 2010) zur theoretischen Rahmung von Unterrichtswirkungen etabliert (Lipowsky 2009). Bildungswirkungen werden demnach von der Angebotsqualität des Unterrichts, der Nutzungsqualität der Lernenden sowie der Synchronisationsqualität zwischen Angebot und Nutzung beeinflusst, wobei der Unterricht in wechselwirkender Beziehung zu kontextuellen Faktoren steht. Wird Lernen aus konstruktivistischer Sicht als individueller, selbstregulierter und ko-konstruktiver Prozess verstanden, so bedeutet dies vor dem Hintergrund einer heterogenen Klassenzusammensetzung, dass für eine optimale Synchronisationsqualität das Angebot passend zu den individuellen Voraussetzungen und Bedürfnissen der Lernenden sein muss. Dazu kann die Lehrperson aufgrund ihrer diagnostischen Einschätzung individuelle Ziele und Inhalte für die Lernenden auswählen oder sie stellt ein offeneres Wahlangebot zur Verfügung, wobei die Schüler*innen selbst Aufgaben, Vorgehensweisen und Unterstützungsangebote auswählen, welche optimal ihren Voraussetzungen und Bedürfnissen entsprechen (Bohl et al. 2011; Klieme und Warwas 2011). Beschränkte Ressourcen der Lehrperson, geforderte Mitsprachemöglichkeiten der Lernenden und das Ziel des selbstständigen Lernens sprechen dabei für eine Unterrichtsöffnung (Dumont 2019; Reusser et al. 2013). Eine Unterrichtsöffnung wird zudem durch Studien unterstützt, die zeigen, dass sich wahrgenommene Wahl- und Mitbestimmungsmöglichkeiten positiv auf das Selbstbestimmungsempfinden auswirken, was wiederum die Motivationsqualität günstig beeinflusst (Deci und Ryan 1993; Hartinger 2005; Rakoczy 2006). Empirische Studien zur Wirksamkeit von Wahl- und Mitbestimmungsmöglichkeiten im Unterricht zeigen jedoch ein uneinheitliches Bild: Je nach Studie finden sich positive, negative oder keine Effekte auf Leistung und Motivation (Evans und Boucher 2015; Katz und Assor 2007). 
Katz und Assor (2007) erklären die heterogene Befundlage anhand der Selbstbestimmungstheorie, welche postuliert, dass die Entwicklung intrinsischer Motivation die Befriedigung der drei Grundbedürfnisse nach Autonomie, Kompetenz und sozialer Eingebundenheit bedingt (Deci und Ryan 1993). Autonomiespielräume sind demnach nur dann lernförderlich, wenn sich die Lernenden bei der Entscheidung kompetent fühlen. Überfordernde Autonomiespielräume können hingegen die Motivation negativ beeinflussen (Furtak und Kunter 2012; Katz und Assor 2007). Vor diesem Hintergrund kommt der Lehrperson in der Ermöglichung eines bedeutungsvollen, anforderungsniveauangepassten und strukturierten Wahlangebots sowie eines Klimas der gegenseitigen Akzeptanz eine zentrale Rolle zu (Assor et al. 2002; Furtak und Kunter 2012; Jang et al. 2010; Katz und Assor 2007). Während die motivationspsychologischen Erkenntnisse vielfach aus Experimentalstudien resultieren, findet die Wirkungsweise auch in der pädagogischen Unterrichtsforschung Bestätigung. Studien zeigen, dass nicht die autonomiefördernde Unterrichtsorganisation selbst, sondern deren Umsetzungsqualität auf der prozessbezogenen Tiefenstruktur entscheidend ist (Lipowsky 2002; Reusser 2011). Die Gestaltung der Prozesse auf der Mikroebene hängt stark von der Kompetenz und Unterstützung der Lehrperson $\mathrm{ab}$, beispielsweise wie es ihr gelingt den Unterricht zu strukturieren, die Lernenden mittels Scaffolding zu unterstützen und kognitiv zu aktivieren, Feedback zu geben oder metakognitive Strategien zu fördern (Alfieri et al. 2011; Hattie 2013; Lipowsky 2002; Lipowsky und Lotz 2015). Nebst der Relevanz der Lernunterstützung konnten in der Unterrichtsforschung differentielle Effekte festgestellt werden: So nutzten konzentrationsschwache Grundschulkinder die Lernzeit in offenen Lernsituationen weniger aufgabenbezogen als konzentrationsstarke Schüler*innen (Lipowsky 1999). Auch starke und schwache Lernende unterschieden sich, da bei wenig Vorwissen das Arbeitsgedächtnis durch die komplexen Aufgabenstellungen zu stark beansprucht und dadurch der Lernprozess erschwert wurde. Nebst dem Vorwissen setzt die produktive Nutzung einer offeneren Lernumgebung nach Lipowsky und Lotz (2015) auch ausreichend Selbststeuerungsfähigkeiten und Motivation voraus. Schwache Lernende sind daher besonders auf ausreichende Strukturierung von Wahlangeboten und auf die Unterstützung der Lehrpersonen angewiesen (Lipowsky und Lotz 2015).

\section{Fragestellung}

Trotz fehlender Definition wird im internationalen Diskurs die Bedeutung von Wahlund Mitbestimmungsmöglichkeiten bzw. choice and voice im Rahmen personalisierter Lernkonzepte deutlich (vgl. Abschn. 2.1 und 2.2). Vor dem Hintergrund der ELFReform (vgl. Abschn. 2.3) kann davon ausgegangen werden, dass Wahl- und Mitbestimmungsmöglichkeiten auch in innovativen Deutschschweizer Schulen, die sich an personalisierten Lernkonzepten orientieren, zentrale Konzeptdimensionen darstellen. Empirische Untersuchungen zum Verständnis und zur praktischen Umsetzung personalisierter Lernkonzepte fehlen jedoch weitgehend. Es interessiert daher, wie die zentralen Aspekte der Wahl- und Mitbestimmungsmöglichkeiten von den Schüler*innen der Sekundarstufe I wahrgenommen werden und ob die Übereinstimmung 
der Einschätzungen auf eine personalisierte Schulkultur hinweist (Fragestellung 1). Vor diesem Hintergrund wird geprüft, ob sich ein Modell mit den Konstrukten Wahlund Mitbestimmungsmöglichkeiten sowohl für die Individual- als auch die Schulebene bilden lässt (Fragestellung 2). Da von einer vielfältigen Praxisumsetzung auszugehen ist, wird weiter untersucht, ob sich das von den Lehrpersonen eingeschätzte Ausmaß der Orientierung an personalisierten Lernkonzepten (Personalisierungsgrad) in der Wahrnehmung der Lernenden spiegelt und sich die Dimensionen choice and voice somit als empirisch bedeutsam für personalisierte Lernkonzepte erweisen (Fragestellung 3). Wie im Abschn. 2.4 dargelegt wurde, sind erweiterte Freiräume nur in Kombination mit adaptiver Lernunterstützung sinnvoll. Daher wird bei allen Fragestellungen ergänzend die Lernunterstützung durch die Lehrperson aus Sicht der Schüler*innen ausgewertet. Für den vorliegenden Artikel sind damit folgende Fragestellungen leitend:

1. Wie nehmen Schüler*innen aus Schulen mit personalisierten Lernkonzepten Wahlund Mitbestimmungsmöglichkeiten (choice and voice) sowie die Lernunterstützung durch die Lehrperson wahr und wie reliabel sind die Schüler*innenwahrnehmungen auf Schulebene?

2. Lässt sich ein Messmodell mit den Konstrukten Wahl- und Mitbestimmungsmöglichkeiten (choice and voice) sowie der Lernunterstützung durch die Lehrperson für die Individual- und die Schulebene bilden?

3. In welchem Maße lässt sich die Schüler*innenwahrnehmung von Wahl- und Mitbestimmungsmöglichkeiten und der Lernunterstützung durch die Lehrperson auf Schulebene durch den von den Lehrpersonen berichteten Personalisierungsgrad erklären?

\section{Methode}

\subsection{Die perLen-Studie als Rahmen, Studiendesign und Stichprobe}

Der vorliegende Artikel basiert auf Daten eines Subsamples der perLen-Studie, welche Schulen mit personalisierten Lernkonzepten (nachfolgend perLen-Schulen genannt) im Hinblick auf Unterrichtsprozesse und -wirkungen sowie die dadurch neu entstandenen Rollen und Herausforderungen für Lehrende und Lernende untersucht (vgl. Stebler et al. 2018). Die nicht repräsentative Gesamtstichprobe umfasst 65 Schulen der Primar- und Sekundarstufe I aus insgesamt 15 Kantonen der Deutschschweiz. Die Schulen wurden im Gesamtprojekt längsschnittlich (2012-2015), mehrperspektivisch und multimethodisch untersucht. Die Sicht der Sekundarschüler*innen wurde in der 7. Klasse (t1), 8. Klasse (t2) und 9. Klasse (t3) mittels Online-Fragebogen erfasst, wobei einzelne Konstrukte nicht zu allen Zeitpunkten erhoben wurden. Wahl- und Mitbestimmungsmöglichkeiten wurden vorwiegend im Onlinefragebogen $\mathrm{t} 2$ erfragt, weshalb sich die vorliegenden Auswertungen darauf stützen.

Von den 40 Sekundarschulen der Gesamtstichprobe nahmen 35 Schulen (davon 5 Privatschulen) an der Online-Befragung t2 mit insgesamt 1046 Schüler*innen der 8. Klasse teil. Die Befragung wurde während der Unterrichtszeit im Juni/Juli 
2014 durchgeführt. Von den befragten Schüler*innen waren 47,2\% weiblich. Durchschnittlich waren die Lernenden 14,9 Jahre alt.

\subsection{Instrumente}

Der Fragebogen für die Schüler*innen beinhaltete sowohl neuentwickelte Skalen und Items als auch solche aus erprobten Instrumenten, wobei aus ökonomischen Gründen nicht immer alle Items einer bestehenden Skala übernommen wurden. Die Items wurden für die perLen-Studie teilweise adaptiert. Zur Erfassung der verschiedenen Aspekte von Wahl- und Mitbestimmungsmöglichkeiten sowie der Lernunterstützung wurden Items aus fünf Originalskalen (Bos et al. 2010; Hagenauer 2011; Helm 2014; Rakoczy et al. 2005; Ramm et al. 2006) eingesetzt. Aufgrund der bestehenden inhaltlichen Überschneidungen der Originalskalen sowie zum Zweck der Optimierung der Messmodelle, wurde in einem ersten Schritt die Dimensionalität mittels explorativer Faktorenanalyse geklärt. Dabei liessen sich drei Faktoren identifizieren, welche den Konstrukten Wahlfreiheit, Mitbestimmung und Lernunterstützung zugeordnet werden konnten. Der erste Faktor Wahlfreiheit umfasste sämtliche Items der Originalskala von Helm (2014), welche mittels fünf Items nach der Häufigkeit der eigenständigen Wahlmöglichkeiten der Schüler*innen in organisatorischen und inhaltlichen Belangen fragt. Auf den zweiten Faktor Mitbestimmung luden sechs Items aus zwei unterschiedlichen Originalskalen: Davon fokussieren drei der Items, inwiefern die Wünsche der Lernenden für die inhaltliche Themenwahl berücksichtigt werden (Ramm et al. 2006) und drei Items erfragen, in welchem Ausmass die Lernenden in Bezug auf persönliche Lernziele involviert werden (Rakoczy et al. 2005). Der dritte Faktor umfasste sieben Items zur Lernunterstützung, welche den Instrumenten zum Unterstützungsverhalten (Bos et al. 2010) und der wahrgenommenen Fürsorglichkeit der Lehrperson (Hagenauer 2011) entnommen wurden. Die Reliabilitätsanalyse zeigte für alle Skalen eine zufriedenstellende bis hohe interne Konsistenz. In Tab. 1 sind die Skalen im Überblick dargestellt. Die einzelnen Items sind im Onlinematerial $1 \mathrm{zu}$ finden.

Tab. 1 Überblick der manifesten Skalen

\begin{tabular}{|c|c|c|c|}
\hline $\begin{array}{l}\text { Skala } \\
\text { Anzahl Items }\end{array}$ & $\begin{array}{l}\text { Beispielitem } \\
\text { Quelle }\end{array}$ & $M(S D)^{\mathrm{c}}$ & $\alpha$ \\
\hline $\begin{array}{l}\text { Wahlfreiheit } \\
5\end{array}$ & $\begin{array}{l}\text { In der Schule entscheide ich selbst, mit wem ich lerne. } \\
\text { Helm (2016) }\end{array}$ & $\begin{array}{l}3,22 \\
(0,62)\end{array}$ & 0,75 \\
\hline $\begin{array}{l}\text { Mitbestimmung } \\
6\end{array}$ & $\begin{array}{l}\text { Im Unterricht legt jede Schülerin, jeder Schüler mit den } \\
\text { Lehrpersonen zusammen die persönlichen Lernziele fest. }^{\text {a }} \\
\text { Ramm et al. (2006), Rakoczy et al. (2005) }\end{array}$ & $\begin{array}{l}2,66 \\
(0,66)\end{array}$ & 0,80 \\
\hline $\begin{array}{l}\text { Lernunterstützung } \\
7\end{array}$ & $\begin{array}{l}\text { Meine Lehrpersonen nehmen sich Zeit, um mir Dinge zu } \\
\text { erklären, die ich nicht verstanden habe. } \\
\text { Bos et al. (2010), Hagenauer (2011) }\end{array}$ & $\begin{array}{l}3,14 \\
(0,55)\end{array}$ & 0,86 \\
\hline
\end{tabular}

\footnotetext{
${ }^{a}$ Antwortformat: häufig (4), manchmal (3), selten (2), nie (1)

bAntwortformat: stimmt genau (4), stimmt eher (3), stimmt eher nicht (2), stimmt gar nicht (1)

${ }^{\mathrm{c}} N=1046$
} 


\subsection{Auswertungsverfahren}

Zur Beantwortung der ersten Forschungsfrage wurden die manifesten Skalen Wahlfreiheit, Mitbestimmung und Lernunterstützung deskriptiv ausgewertet. Zur Überprüfung, ob die Schulhausebene in der Auswertung zu berücksichtigen ist bzw. ob eine Zusammenfassung der Daten auf Schulhausebene reliabel ist, wurden die Intraklassenkorrelationen (ICC1 und ICC2) für die Schulebene berechnet ${ }^{1}$. Der ICC1 gibt an, welcher Anteil der Gesamtvarianz auf Unterschiede zwischen den Schulen zurückgeführt werden kann während der ICC2 eine Aussage über die Reliabilität der Aggregatdaten erlaubt (Gärtner 2010). Die deskriptiven Auswertungen sowie die Berechnung der Intraklassenkorrelationen erfolgten mit der Statistiksoftware SPSS 27 (IBM Corp 2020).

Zur Überprüfung, ob sich ein Messmodell mit den Konstrukten Wahl- und Mitbestimmungsmöglichkeiten sowie der Lernunterstützung für die Individual- und die Schulebene bilden lässt (zweite Forschungsfrage), wurde mittels konfirmatorischer Mehrebenen-Faktorenanalyse (Multilevel Confirmatory Factor Analysis [MCFA]; Ebene 1: Schüler*innen, Ebene 2: Schulen) die Faktorstruktur der vorgängig manifest gebildeten Skalen mit dem robusten MLR-Schätzer überprüft. Da zahlreiche Indikatoren pro Faktor zu einem geringeren Anteil geteilter Varianz und zu einer grossen Anzahl zu schätzender Parameter führen, wurde durch das Zusammenziehen von Indikatoren zu Parcels das Modell entlastet, ohne dass die Nachteile der Kontrolle zufälliger Messfehler aufgegeben werden musste (Marsh et al. 2013; Rioux et al. 2020). Die Überprüfung der Dimensionalität erfolgte mittels Schätzung und Modellvergleich mehrerer MCFAs mit unterschiedlicher Faktorenzahl. Die MCFA wurde anschließend mit dem Step-Up-Ansatz auf cross-level-Messinvarianz überprüft, wobei zunehmend restriktivere Modelle gebildet wurden und anhand des Chi ${ }^{2}$ Differenztestes überprüft wurde, ob sich diese signifikant vom Ausgangsmodell unterscheiden (Kleinke et al. 2017).

Zur Überprüfung, ob sich die Unterschiede in der mittleren Wahrnehmung der Lernenden zwischen den Schulen im Hinblick auf Wahlfreiheit und Mitbestimmung durch den von den Lehrpersonen eingeschätzten schulbezogenen Personalisierungsgrad erklären lassen (dritte Forschungsfrage), wurde ausgehend vom beschriebenen Messmodell ein Mehrebenen-Strukturgleichungsmodell (Multilevel Structural Equation Modeling [MSEM]) geschätzt. Für das Mehrebenen-Strukturgleichungsmodell wurde die Prädiktorvariable „Personalisierungsgrad“ in manifester Form auf Schulebene ins Modell aufgenommen. Die Bildung des Personalisierungsgrades wird im folgenden Kapitel beschrieben. Das Messmodell sowie auch das Mehrebenen-Strukturgleichungsmodell wurden mit Mplus, Version 8 (Muthén und Muthén 2017) geschätzt. Die Beurteilung der Modelle wurde nach Empfehlung von Byrne (2012) anhand der Fit-Werte $\chi^{2}$, CFI (Comparative Fit Index), RMSEA (Root Mean Square

\footnotetext{
1 Viele perLen-Schulen führen anstelle traditioneller Klassen flexibel zusammengesetzte Lerngruppen. Daher und vor dem Hintergrund, dass sich personalisierte Lernkonzepte in der Regel auf die ganze Institution beziehen, wird im vorliegenden Artikel die Schule als Aggregationsebene verwendet. Aufgrund unterschiedlicher Schulgröße und Konzeptumsetzung variiert die Anzahl befragter Schüler*innen pro Schule zwischen 7 und 74 .
} 
Error of Approximation) und SRMR (Standardized Root Mean Residual) dokumentiert, wobei sich die Beurteilung an den Cutoff-Werten von Hu und Bentler (1999) orientierte. Demnach sollte der CFI mindestens 0,95 betragen, der RMSEA nicht über 0,06 und der SRMR nicht über 0,08 liegen.

\subsection{Personalisierungsgrad}

Die perLen-Stichprobe ist bezüglich Umsetzung personalisierter Lernkonzepte heterogen. Zur Strukturierung der Stichprobe wurde in der perLen-Forschungsgruppe die Variable „Personalisierungsgrad“ gebildet. Der Wert, welcher für jede Schule bestimmt wurde, setzt sich aus den gemittelten Einschätzungen der Lehrpersonen und Schulleitungen auf die Frage ,Wie stark orientiert sich Ihre Schule an personalisierten Lernkonzepten?" der Online-Befragungen t2 und t3 zusammen (für weiterführende Erläuterungen siehe Stebler et al. 2018). Das Ausmaß wurde auf einer vierstufigen Skala von 1 (kaum) bis 4 (sehr stark) eingeschätzt. Der mittlere Wert des Personalisierungsgrades für die 35 Sekundarschulen der Substichprobe lag bei 3,11 $(S D=0,44)$ bei einer Spannweite von 2,4 (Min. =1,60, Max. =4,00).

\section{Ergebnisse}

\subsection{Wahrnehmung der Schüler*innen von choice and voice}

Die deskriptive Auswertung der Skalen zeigte, dass die perLen-Schulen aus Sicht der Schüler*innen Wahlmöglichkeiten (choice) gewährten $(M=3,22, S D=0,62)$. Die Analyse der Einzelitems wies darauf hin, dass vorwiegend Wahlmöglichkeiten bezüglich Zeiteinteilung $(M=3,57, S D=0,71)$ und Sozialform $(M=3,31, S D=0,80)$ wahrgenommen wurden. So gaben $68 \%$ der befragten Schüler*innen an, dass sie in der Schule häufig selbst die Zeit zum Lernen einteilen können. Lediglich 8,4\% der Lernenden gab an, dass sie ihre Zeiteinteilung nie $(2,2 \%)$ oder selten $(6,2 \%)$ selbst bestimmen können. Am wenigsten nahmen die Schüler*innen inhaltliche Wahlmöglichkeiten $(M=3,04, S D=1,00)$ und Wahlmöglichkeiten bezüglich des Lernortes $(M=3,04, S D=0,90)$ wahr. Die hohen Standardabweichungen wiesen auf eine substantielle Variation der Wahrnehmung hin. Mitbestimmungsmöglichkeiten (voice) wurden signifikant weniger häufig wahrgenommen $(M=2,66, S D=0,66$; $t(1045)=169,130, p<0,001)$. Auswertungen auf Ebene der Einzelitems zeigten, dass die höchste Zustimmung das Item „Im Unterricht vereinbaren wir mit unseren Lehrpersonen unsere persönlichen Lernziele“ $(M=2,90, S D=0,86)$ erzielte. Die Schüler*innen der perLen-Schulen nahmen die Lernunterstützung durch die Lehrperson insgesamt positiv wahr $(M=3,14, S D=0,55)$. Die Mittelwerte und Standardabweichungen sämtlicher Einzelitems sind im Onlinematerial 1 aufgeführt.

Zur Klärung, ob von einer personalisierten Schulkultur gesprochen werden kann, wurde zudem die Reliabilität der Schüler*innenwahrnehmung untersucht. Die ICC1 für Mitbestimmung und Wahlfreiheit wiesen auf eine ausreichende Variabilität zwischen den Schulen hin und bedeuten, dass sich 18\% (Mitbestimmung) bzw. 17\% (Wahlfreiheit) der Varianz in der Wahrnehmung der Lernenden durch die Schulen 
erklären lassen, was nach Lebreton und Senter (2008) einem mittleren Effekt entspricht. Die Aggregation für die beiden Skalen auf Schulebene wurde aufgrund des ICC2 als reliabel eingeschätzt und betrug für die Skala Mitbestimmung 0,87 und für die Skala Wahlfreiheit 0,86. Bezüglich Wahrnehmung der Lernunterstützung aus Lernendensicht zeigte der ICC1 nur einen kleinen Schuleffekt von $6 \%$ (Lebreton und Senter 2008). Die Reliabilität des ICC2 von 0,64 kann als knapp genügend eingeschätzt werden, wobei Lebreton und Senter (2008) darauf hinweisen, dass der häufig verwendete Grenzwert von 0,70 für den ICC2 nicht als starre, sondern heuristische Regel betrachtet werden soll. Aufgrund der Auswertungen ist eine Aggregation der Skalen auf Schulebene möglich und eine Berücksichtigung der Clusterung der Daten notwendig.

\subsection{Bildung eines dreidimensionalen, hierarchischen Messmodells}

Für das Messmodell wurden in einem ersten Schritt Parcels gebildet (vgl. Abschn. 5.2.1). In einem zweiten Schritt wurde die Passung der dreidimensionalen Struktur anhand von Alternativmodellen überprüft (vgl. Abschn. 5.2.2) und in einem dritten Schritt folgte die Überprüfung der cross-level-Messinvarianz (vgl. Abschn. 5.2.3).

\subsubsection{Bildung homogener Parcels}

Auf Empfehlung von Marsh et al. (2013) wurden homogene Parcels gebildet, bei welchen die Binnenstrukturen nicht überdeckt werden. Da die Skalen Mitbestimmung und Lernunterstützung Items aus verschiedenen Originalskalen umfassten (vgl. Abschn. 4.2), wurden je die Items einer Originalskala zu einem Parcel zusammengefasst. Die Skala Wahlfreiheit wurde als Originalskala übernommen, weshalb die Items nach inhaltlichen Kriterien (organisatorische versus inhaltliche Wahlfreiheit) aufgeteilt wurden. Die gebildeten Parcels sind in Tab. 2 zusammengefasst.

Tab. 2 Überblick der Parcels

\begin{tabular}{lll}
\hline Parcel & Inhalt (Quelle) & Anzahl Items \\
\hline Wahlfreiheit & & \\
Par_W1 & Organisatorische Wahlfreiheit (Helm 2016) & 3 \\
Par_W2 & Inhaltliche Wahlfreiheit (Helm 2016) & 2 \\
Mitbestimmung & \\
Par_M1 & Gemeinsame Zielvereinbarung (Rakoczy et al. 2005) & 3 \\
Par_M2 & Mitwirken bei der Stoffauswahl (Ramm et al. 2006) & 3 \\
Lernunterstützung & \\
Par_L1 & Lernunterstützung durch die Lehrperson (Bos et al. 2010) \\
Par_L2 & Fürsorglichkeit der Lehrperson (Hagenauer 2011) & 4 \\
\hline
\end{tabular}


Tab. 3 Zusammenfassung der Fit-Werte der MCFA-Modelle mit MLR-Schätzer

\begin{tabular}{llllll}
\hline $\begin{array}{l}\text { Modell: Modellbeschrei- } \\
\text { bung }\end{array}$ & $\chi^{2}(\mathrm{df})$ & CFI & RMSEA & SRMR $^{\mathrm{a}}$ & BIC \\
\hline M1: 1 Faktor & $614,998(20)$ & 0,651 & 0,169 & $0,133 / 0,090$ & 11462,460 \\
M2: 2 Faktoren $^{\mathrm{b}}$ & $314,248(18)$ & 0,826 & 0,125 & $0,088 / 0,081$ & 11190,512 \\
M3: 3 Faktoren $^{\mathrm{c}}$ & $24,480(14)$ & 0,994 & 0,027 & $0,016 / 0,089$ & 10972,499 \\
\hline
\end{tabular}

${ }^{a}$ Der SRMR-within-Wert wird vor, der SRMR-between-Wert nach dem Schrägstrich angegeben

${ }^{\mathrm{b}}$ Faktor 1: Wahlfreiheit und Mitbestimmung; Faktor 2: Lernunterstützung

${ }^{\mathrm{c}}$ Faktor 1: Wahlfreiheit; Faktor 2: Mitbestimmung; Faktor 3: Lernunterstützung

\subsubsection{Konfirmatorische Mehrebenen-Faktorenanalyse}

Zur Überprüfung der Modellpassung wurden zwei alternative MCFAs mit einfaktorieller (M1) bzw. zweifaktorieller (M2) Struktur getestet. Beide Alternativmodelle wiesen eine mangelnde Passung zu den Daten auf, während die dreifaktorielle MCFA (M3) eine angemessene Modellpassung für die mittels Parcels latent modellierten Faktoren Wahlfreiheit, Mitbestimmung und Lernunterstützung auf beiden Ebenen zeigte (vgl. Tab. 3). Aufgrund negativer Varianzen wurden auf der Schulebene zwei Residuuen auf null fixiert (Kleinke et al. 2017). In der dreifaktoriellen MCFA waren sämtliche standardisierte Ladungen auf beiden Ebenen statistisch signifikant ( $p<0,001)$ und größer als 0,54 (Individualebene) bzw. 0,75 (Schulebene). Die Faktorkorrelationen waren auf der Schulebene bedeutend höher, weshalb sich die Frage der Diskriminanzvalidität der Konstrukte auf Ebene 2 stellte (vgl. Onlinematerial 2). Aufgrund der Fit-Werte, insbesondere des für den Vergleich nicht verschachtelter Modelle zentralen informationstheoretischen Masses des BIC, und zugunsten der inhaltlichen Interpretierbarkeit (Kleinke et al. 2017) wurde das Modell M3, mit dreifaktorieller Struktur auf beiden Ebenen, favorisiert.

\subsection{3 Überprüfung auf cross-level-Messinvarianz}

Aufgrund des Modellfits der dreifaktoriellen MCFA konnte konfigurale Invarianz angenommen werden (Kleinke et al. 2017). Zur Überprüfung der cross-level metrischen Invarianz wurden im Modell 4 (M4) jeweils die sich entsprechenden Faktorladungen über beide Analyseebenen gleichgesetzt. Der für MLR-Schätzer angepasste $\chi^{2}$ Differenztest zeigte für das restringierte Modell M4 keinen signifikant schlechteren

Tab. 4 Überprüfung auf cross-level-Messinvarianz

\begin{tabular}{lllllll}
\hline $\begin{array}{l}\text { Modell: } \\
\text { Modellbeschreibung }\end{array}$ & $\chi^{2}(\mathrm{df})$ & CFI & RMSEA SRMR $^{\mathrm{a}}$ & BIC & $\Delta \chi^{2 \mathrm{~b}}$ \\
\hline M3: 3 Faktoren & $\begin{array}{l}24,480 \\
(14)\end{array}$ & 0,994 & 0,027 & $0,016 / 0,089$ & 10972,499 & - \\
& & & & & \\
$\begin{array}{l}\text { M4: Test auf metrische } \\
\text { cross-level-Invarianz }\end{array}$ & $\begin{array}{l}29,828 \\
(17)\end{array}$ & 0,992 & 0,027 & $0,017 / 0,080$ & 10957,714 & $\begin{array}{l}\Delta \chi^{2}=5,32, \\
\mathrm{df}=3, p=0,149\end{array}$ \\
$\begin{array}{l}\text { M5: Test auf skalare } \\
\text { cross-level-Invarianz }\end{array}$ & $\begin{array}{l}182,622 \\
(21)\end{array}$ & 0,905 & 0,086 & $0,029 / 0,115$ & 11007,185 & $\begin{array}{l}\Delta \chi^{2}=1439,76, \\
\mathrm{df}=7, p<0,000\end{array}$ \\
\hline
\end{tabular}

aDer SRMR-within-Wert wird vor, der SRMR-between-Wert nach dem Schrägstrich angegeben ${ }^{\mathrm{b}} \mathrm{Chi}^{2}$-Differenztest mit Satorra-Bentler-Korrektur bei Vergleich mit Modell M3 
Modellfit im Vergleich zum Modell M3, weshalb metrische Invarianz angenommen werden konnte (Satorra-Bentler corrected $\Delta \chi^{2}=5,32$, df $=3$, n. s.) (vgl. Tab. 4). Im Modell 5 (M5) wurde cross-level skalare Invarianz durch das Restringieren der Fehlervarianzen auf null überprüft, wobei sich der Modellfit signifikant verschlechterte. Für das Mehrebenen-Strukturgleichungsmodell wurde deshalb das ebenenbezogen metrisch invariate Messmodell M4 verwendet (vgl. Onlinematerial 3). Die ICC1Werte der latenten Faktoren Wahlfreiheit und Mitbestimmung betrugen im Modell M4 je 0,16, der ICC1-Wert des Faktors Lernunterstützung 0,04. Damit waren sie nahezu gleich stark ausgeprägt wie die zuvor berichteten ICC1-Werte der manifesten Skalen.

\subsection{Schüler*innenwahrnehmung in Abhängigkeit des Personalisierungsgrades}

Im Rahmen der dritten Forschungsfrage wurde mit einem Mehrebenen-Strukturgleichungsmodell untersucht, in welchem Maße sich die Schüler*innenwahrnehmung von Wahl- und Mitbestimmungsmöglichkeiten sowie der Lernunterstützung auf Schulebene durch den von den Lehrpersonen berichteten Personalisierungsgrad erklären lassen. Abb. 1 zeigt das auf der MCFA M4 aufbauende Modell mit

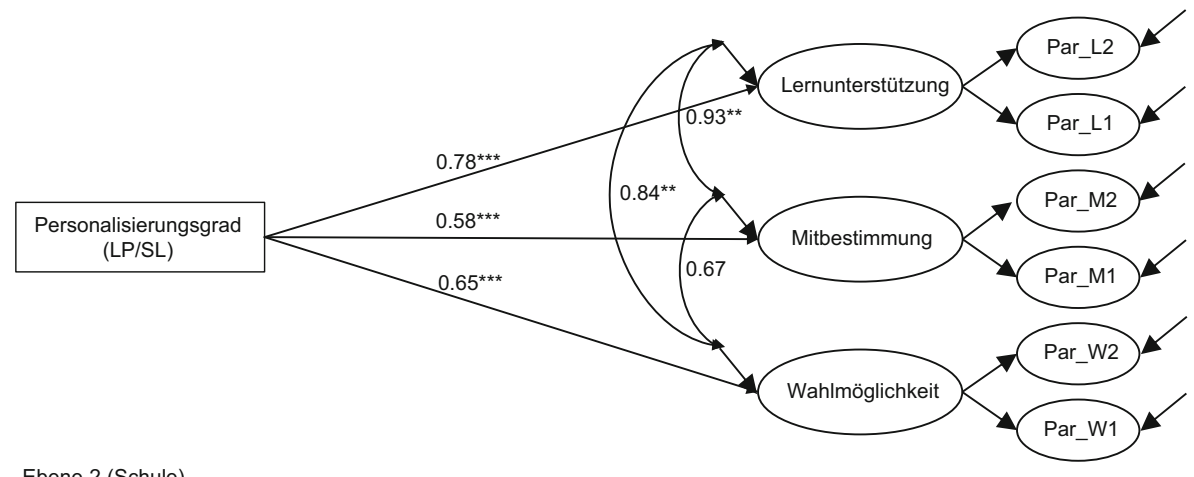

(Schule)

Ebene 1 (Schüler/innen)

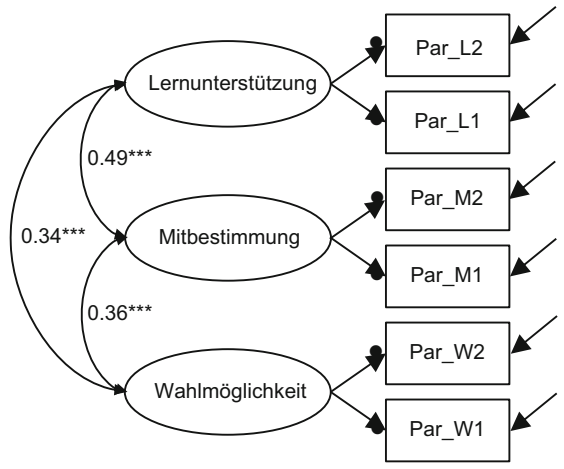

Abb. 1 Mehrebenen-Strukturgleichungsmodell mit standardisierten Regressionskoeffizienten und Korrelationen. ${ }^{* *} p<0,001,{ }^{* *} p<0,01, * p<0,05$; $\mathrm{N}_{\text {within: }}$ 1046, $\mathrm{N}_{\text {between }}$ : 35; Personalisierungsgrad (LP/SL): Personalisierungsgrad eingeschätzt durch Lehrer*innen und Schulleiter*innen 
den standardisierten Koeffizienten und Korrelationen. Die Modellpassung ist gut $\left(\chi^{2}(20)=37,222, \mathrm{CFI}=0,991 ; \mathrm{RMSEA}=0,029 ; \mathrm{SRMR}_{\text {within }}=0,017 ; \mathrm{SRMR}_{\text {between }}=\right.$ $0,076)$.

Der von den Lehrpersonen berichtete Personalisierungsgrad erwies sich als statistisch bedeutsamer Prädiktor für die von den Lernenden auf Schulebene wahrgenommene Wahlfreiheit $(\beta=0,65, p<0,001)$, Mitbestimmung $(\beta=0,58, p<0,001)$ und Lernunterstützung $(\beta=0,78, p<0,001)$. Auf der Individualebene korrelierten alle drei Konstrukte signifikant und mit mittlerer Stärke miteinander. Auf Schulebene zeigten sich starke Korrelationen zwischen der aus Lernendensicht wahrgenommenen Unterstützung der Lehrperson mit der Wahlfreiheit $(r=0,84, p<0,01)$ und Mitbestimmung $(r=0,93, p<0,01)$. Mitbestimmung und Wahlfreiheit korrelierten auf der Schulebene zwar nominell stark $(r=0,67)$, jedoch nicht statistisch signifikant miteinander.

\section{Zusammenfassung und Diskussion}

Der facettenreiche Sammelbegriff „Personalisiertes Lernen“ beinhaltet vielfältige pädagogische Konzepte und Massnahmen, die den Anspruch verfolgen, Lernarrangements an den persönlichen Bedürfnissen aktiv lernender Schüler*innen auszurichten. Mit dem Slogan choice and voice werden dabei Wahlfreiheiten sowie ein aktives Einbringen und Mitbestimmen der Lernenden gefordert. Das in der Literatur vorfindbare empirische Gesamtbild über Wirkungen von Wahl- und Mitbestimmungsmöglichkeiten ist dabei uneinheitlich, was unter anderem auf unterschiedliche Verständnisse und Umsetzungsmöglichkeiten von autonomiefördernden Unterrichtskonzepten zurückzuführen ist. Was verschiedene Studien zeigen konnten, ist, dass auch (und ganz besonders) im offenen Unterricht nicht die sichtbaren Organisationsstrukturen, sondern die Qualität der Lehr-Lernprozesse auf der Ebene ihrer Tiefenstruktur für den Lernertrag entscheidend sind, dabei (mit Bezug auf die Lernvoraussetzungen der Schüler*innen) differentielle Effekte zu erwarten sind und der Lernunterstützung durch die Lehrperson eine grosse Bedeutung zukommt (Alfieri et al. 2011; Bohl et al. 2011; Lipowsky 2002; Reusser 2011). In der Deutschschweiz schließt die Forderung nach choice and voice an frühere Unterrichtsreformen an, welche den Schüler*innen einen passgenaueren Unterricht und selbstbestimmteres Lernen ermöglichen sollten. Obwohl verschiedene innovative Schulen der Deutschschweiz sich an personalisierten Lernkonzepten orientieren und langjährige Praxiserfahrungen mit Wahl- und Mitbestimmungsmöglichkeiten haben, ist wenig über die Verbreitung und Wahrnehmung dieser Autonomiespielräume aus Lernendensicht bekannt. Der Artikel leistet einen Beitrag zur Schließung der Forschungslücke.

Mit der ersten Forschungsfrage wurde untersucht, wie Schüler*innen in Schulen, die sich an personalisierten Lernkonzepten orientieren, Wahl- und Mitbestimmungsmöglichkeiten sowie die Lernunterstützung durch die Lehrperson wahrnehmen und ob die Einschätzung auf Schulebene als reliabel betrachtet werden kann. Die Ergebnisse zeigen, dass die Lernenden der Sekundarstufe I im Unterricht regelmäßig Wahlmöglichkeiten wahrnehmen, wobei insgesamt eine höhere Entscheidungsfreiheit bei organisatorischen Fragen wahrgenommen wird. Aus Sicht der Schüler*innen sind 
Wahlmöglichkeiten in Bezug auf Lernort und Inhalt weniger häufig. Während sich das geringere Ausmaß der Wahlfreiheit mit Bezug auf den Lernort durch beschränkte räumliche und personelle Ressourcen erklären lässt, ist die Wahrnehmung in Bezug auf inhaltliche Wahlmöglichkeiten vor dem Hintergrund verbindlicher Lehrplanvorgaben plausibel. Im Kontext eines institutionalisierten Lernsettings und mit Blick auf bisherige Untersuchungen (Müller-Kuhn et al. 2020) kann die Wahrnehmung der Wahlfreiheit aus Lernendensicht als positiv eingeschätzt werden. Das gemeinsame Aushandeln von Zielen und Inhalten (Mitbestimmung) wird von den Schüler*innen weniger positiv wahrgenommen. Eine mögliche Erklärung dafür ist, dass viele Sekundarschulen aus der perLen-Stichprobe ihren Unterricht in einem Zusammenspiel von Inputlektionen und Lernlandschaften ${ }^{2}$ organisieren. Während die Inputlektionen mit einem geführten Klassenunterricht vergleichbar sind, bieten die Lernlandschaften ein Gefäß für individuelle Lernzeit, welche in weitgehender Selbstverantwortung der Schüler*innen genutzt wird. Es findet somit weniger ein gemeinsames Planen, Aushandeln und Mitbestimmen statt, sondern die Schüler*innen können in klar vorgegebenen Zeitfenstern selbstbestimmt bzw. selbstorganisiert lernen, was die höhere Einschätzung der Wahlfreiheit erklärt. Die positive Einschätzung der Lernunterstützung zeigt, dass sich die Schüler*innen gut von den Lehrpersonen unterstützt fühlen. Die Auswertungen zur Wahrnehmung der Wahl- und Mitbestimmungsmöglichkeiten aus Lernendensicht wurden auf Basis der Individualdaten ohne Berücksichtigung der Schulebene ausgewertet. Da sich die Anzahl der befragten Schüler*innen pro Schule stark unterscheidet, fallen große Schulen stärker ins Gewicht, was zu Verzerrungen führen könnte. Eine Replikation der Analysen basierend auf Daten, die auf Schulebene aggregiert wurden, ergab jedoch weitgehend identische Ergebnisse. Damit kann eine grobe Verzerrung der Befunde durch ungleiche Schulgrößen ausgeschlossen werden.

Da viele perLen-Schulen keine traditionellen Klassen mehr führen, sondern den Unterricht in flexibel zusammengesetzten Lerngruppen organisieren, wurden die Intraklassenkorrelationen für die Schulebene berechnet. Dabei zeigte sich, dass die Schulebene - trotz unterschiedlichen Lehrpersonen und Lerngruppen - einen substantiellen Anteil der Varianz in der Wahrnehmung der Schüler*innen hinsichtlich Wahl- und Mitbestimmungsmöglichkeiten erklären kann und die Einschätzungen auf Schulebene reliabel sind. Inhaltlich lässt dies darauf schließen, dass die Schulen der perLen-Studie auf diese Aspekte der Personalisierung bezogen breit geteilte Standards in Form einer einheitlichen Schulkultur entwickelt haben, welche die Praxis maßgeblich anleiten und im Schulalltag von den Schüler*innen konsistent wahrgenommen werden. Für die Wahrnehmung der Lernunterstützung zeigt sich nur ein kleiner Schuleffekt. Dies ist nachvollziehbar, da diesbezüglich weniger das Schulkonzept oder die Schulorganisation, sondern stärker die persönliche Beziehung zu den (verschiedenen) Lehrpersonen sowie individuelle Merkmale der Schüler*innen die Einschätzung mitbeeinflussen.

Im Rahmen der zweiten Forschungsfrage wurde die Bildung eines hierarchischen Messmodells für die Konstrukte Wahl- und Mitbestimmungsmöglichkeiten

\footnotetext{
${ }^{2}$ Lernlandschaften werden in den perLen-Schulen auch als Lernatelier, Lernbüro oder Lernoffice bezeichnet.
} 
(choice and voice) sowie der Lernunterstützung überprüft. Da die Cluster-Fallzahl mit 35 Schulen gemessen an der Modellkomplexität klein ist, wurden zur Entlastung des Modells homogene Mittelwert-Parcels gebildet (Marsh et al. 2013; Rioux et al. 2020). Das Parceling wird in der Literatur kontrovers diskutiert (Little et al. 2002, 2013; Marsh et al. 2013). Für den vorliegenden Artikel wurde das Vorgehen als angemessene Auswertungsstrategie zur Modellentlastung eingeschätzt, da von eindimensionalen Konstrukten ausgegangen werden konnte und im Zentrum des Erkenntnisinteressens nicht die einzelnen Items, sondern die latenten Konstrukte standen (Little et al. 2002).

Auffallend bei der MCFA waren die deutlich höheren Faktorkorrelationen auf Schulebene, insbesondere zwischen den wahrgenommenen Mitbestimmungsmöglichkeiten und der wahrgenommenen Unterstützung der Lehrperson. Während aus inhaltlichen Gründen die dreidimensionale Struktur beibehalten wurde, wird der starke Zusammenhang im Rahmen der Diskussion der dritten Forschungsfrage erörtert.

Mit der dritten Forschungsfrage wurde untersucht, inwiefern die Wahrnehmung der Schüler*innen im Hinblick auf Wahl- und Mitbestimmungsmöglichkeiten sowie der Lernunterstützung mit dem von den Lehrpersonen und Schulleitungen eingeschätzten Personalisierungsgrad übereinstimmt bzw. sich auf diesen zurückführen lässt. Das Mehrebenen-Strukturgleichungsmodell bestätigt den Personalisierungsgrad als relevanten Prädiktor: In Schulen, die von den Lehrpersonen und Schulleitungen als stark personalisiert eingeschätzt werden, nehmen die Schüler*innen ausgeprägtere Wahl- und Mitbestimmungsmöglichkeiten wahr. Zugleich schätzen sie auch die Lernunterstützung positiver ein. Während der positive Effekt des Personalisierungsgrades auf die Wahl- und Mitbestimmungsmöglichkeiten aufgrund der Theorie erwartet wurde, erstaunt der starke Einfluss auf die wahrgenommene Lernunterstützung, da sich Schüler*innen unabhängig vom Unterrichtskonzept von den Lehrpersonen gut unterstützt fühlen sollten. Aufgrund der starken Korrelation kann vermutet werden, dass insbesondere den Lehrpersonen aus den sehr stark personalisierten Schulen eine erfolgreiche Konzeptumsetzung, bei welcher die Orientierung an den individuellen Bedürfnissen der Schüler*innen zentral ist, besonders gut gelingt.

Ein weiteres interessantes Ergebnis des Mehrebenen-Strukturgleichungsmodells sind die starken Zusammenhänge zwischen den wahrgenommenen Wahl- und Mitbestimmungsmöglichkeiten und der Lernunterstützung. Schüler*innen, welche häufiger Wahlfreiheiten wahrnehmen und gemeinsam mit der Lehrperson Ziele und Inhalte aushandeln können, fühlen sich insgesamt besser von den Lehrpersonen unterstützt - obwohl autonomiefördernde Lernumgebungen besondere Anforderungen an die Unterstützung der Lehrperson stellen. In didaktischer und lernpsychologischer Perspektive ist bzw. wäre dies ein erfreulicher Befund, gilt doch die Qualität des Lernens bei geöffneten, d.h. erweiterte Autonomiespielräume ermöglichenden Settings als stark von der Qualität der Lernunterstützung abhängig (Alfieri et al. 2011; Lipowsky 2002). Ob aus dem vorliegenden Befund wirklich auf eine überzeugende Lernunterstützungspraxis geschlossen werden kann, oder ob dahinter eher Ausstrahlungseffekte liegen, ist in weiterführenden Auswertungen zu klären. 
Aus theoretischer Perspektive wäre die Korrelation zwischen Wahl- und Mitbestimmungsmöglichkeiten plausibel, da sowohl choice als auch voice relevante Dimensionen personalisierten Lernens sind. Im Mehrebenen-Strukturgleichungsmodell ist jedoch nur auf der Individualebene die Korrelation dieser Konstrukte signifikant. Dieses Ergebnis lässt sich nebst der geringen Clusterzahl auch mit den unterschiedlichen Korrelationen der wahrgenommenen Wahl- und Mitbestimmungsmöglichkeiten zwischen den Schulen erklären. So zeigten ergänzende Auswertungen, dass es Schulen gibt, in denen die Schüler*innen sowohl hohe Wahl- als auch Mitbestimmungsmöglichkeiten wahrnehmen und Schulen in denen Schüler*innen das eine deutlich ausgeprägter als das andere wahrnehmen. Daher wäre für weiterführende Auswertungen ein vertiefter Blick lohnenswert, wobei verschiedene Konfigurationen und Umsetzungen der Wahl- und Mitbestimmungsmöglichkeiten sowie der Lernunterstützung in den einzelnen Schulen untersucht würden. Hierfür speziell geeignet wären ein person- (bzw. hier schul-) zentrierter Ansatz, um solche Konfigurationen in Form von Typen bzw. latenten Klassen zu fassen.

\section{Limitationen}

Bei der Interpretation der Ergebnisse sind auch gewisse Limitationen zu berücksichtigen. So basieren sämtliche Ergebnisse der vorliegenden Untersuchung auf Selbsteinschätzungen. Die an der Studie beteiligten Schulen schätzten sich selbst als personalisiert ein, wobei diese Einschätzung mehr aus dem innovativen Selbstverständnis der Schulen als dem Erfüllen klar definierter Kriterien resultiert haben dürfte. Auch wenn damit der Leitidee Rechnung getragen werden konnte, dass sich personalisierte Lernkonzepte an den jeweiligen Bedürfnissen der Beteiligten orientieren und daher verschiedene Gesichter aufweisen können (Bray und McClaskey 2015), war dies sicher mit ein Grund dafür, dass die perLen-Stichprobe sehr heterogen ausfiel (vgl. auch Stebler et al. 2018). Aufgrund der Stichprobenheterogenität sowie dem Fehlen einer Vergleichsstichprobe bleibt jedoch offen, ob und wie stark sich die innovativen perLen-Schulen im Hinblick auf Wahl- und Mitbestimmungsmöglichkeiten von ,regulären Schulen“, d. h. einer Zufallsstichprobe unterscheiden.

Bei der Unterrichtswahrnehmung aus Schüler*innensicht muss berücksichtigt werden, dass die Einschätzung sowohl von individuellen Personenmerkmalen als auch der Gruppe (Schule) mitbeeinflusst ist, was die Vergleichbarkeit zwischen den Schulen einschränkt. Bei der Wahrnehmung der Lernunterstützung zeigte sich zudem, dass nur ein kleiner Anteil der Gesamtvarianz auf die Schulebene zurückgeführt werden kann. Einschränkend gilt es zudem festzuhalten, dass die vorliegenden Auswertungen keine Aussagen darüber erlauben, inwiefern relevante Personenmerkmale - wie beispielsweise der Leistungsstand - die Wahrnehmung beeinflussen. Da aufgrund der Theorie differentielle Effekte zu erwarten sind, erscheint es angezeigt, in weiterführenden Untersuchungen den Zusammenhang von autonomiefördernden Lernumgebungen und Lernunterstützung unter Berücksichtigung individueller Personenmerkmale zu untersuchen.

Trotz beachtlichem „Verzerrungspotential“ (Wagner 2008, S. 6) der Unterrichtswahrnehmung aus Sicht der Schüler*innen, bleibt diese Datenquelle für die Unterrichtsforschung essentiell: Denn in vielerlei Hinsicht hat sich weniger die „ob- 
jektive Wahrnehmung“ durch Beobachtende, als vielmehr die Wahrnehmung der Schüler*innen als bedeutsam für deren Lernen erwiesen (Bohl und Kucharz 2010; De Jong und Westerhof 2001; Hartinger 2006; Helm 2016; Lüdtke et al. 2009; Pauli et al. 2007). Autonomiespielräume müssen somit nicht nur vorhanden, sondern von den Schüler*innen auch als solche wahrgenommen werden, damit sie genutzt werden und die Schüler*innen sich als selbstbestimmt erleben. Die Auswertungen zeigen, dass dies in den perLen-Schulen in hohem Maße der Fall ist. Ob die Wahrnehmung von choice and voice auch tatsächlich Wirkung entfaltet, muss Gegenstand weiterer Analysen sein.

Der vorliegende Beitrag zeigt, dass es sich bei Wahl- und Mitbestimmungsmöglichkeiten nicht nur aus theoretischer, sondern auch aus empirischer Sicht um eine bedeutsame Dimension personalisierter Lernkonzepte handelt. Für ein tieferes Verständnis von deren Voraussetzungen und Wirkungen sowie für die Einschätzung ihrer Praxisbedeutsamkeit, braucht es künftig weitere qualitative und quantitative Studien, in denen Konfigurationen von Wahl- und Mitbestimmungsmöglichkeiten in Verbindung mit weiteren Merkmalen einer personalisierten Unterrichtsgestaltung in der praktischen Umsetzung untersucht werden.

Zusatzmaterial online Zusätzliche Informationen sind in der Online-Version dieses Artikels (https://doi. org/10.1007/s42010-021-00122-x) enthalten.

Danksagung Die perLen-Studie wurde von der Stiftung Mercator Schweiz gefördert.

Funding Open access funding provided by University of Zurich

Open Access Dieser Artikel wird unter der Creative Commons Namensnennung 4.0 International Lizenz veröffentlicht, welche die Nutzung, Vervielfältigung, Bearbeitung, Verbreitung und Wiedergabe in jeglichem Medium und Format erlaubt, sofern Sie den/die ursprünglichen Autor(en) und die Quelle ordnungsgemäß nennen, einen Link zur Creative Commons Lizenz beifügen und angeben, ob Änderungen vorgenommen wurden.

Die in diesem Artikel enthaltenen Bilder und sonstiges Drittmaterial unterliegen ebenfalls der genannten Creative Commons Lizenz, sofern sich aus der Abbildungslegende nichts anderes ergibt. Sofern das betreffende Material nicht unter der genannten Creative Commons Lizenz steht und die betreffende Handlung nicht nach gesetzlichen Vorschriften erlaubt ist, ist für die oben aufgeführten Weiterverwendungen des Materials die Einwilligung des jeweiligen Rechteinhabers einzuholen.

Weitere Details zur Lizenz entnehmen Sie bitte der Lizenzinformation auf http://creativecommons.org/ licenses/by/4.0/deed.de.

\section{Literatur}

Agostini, E., Schratz, M., \& Risse, E. (2018). Lernseits denken-erfolgreich unterrichten: Personalisiertes Lehren und Lernen in der Schule. Hamburg: AOL-Verlag.

Alfieri, L., Brooks, P. J., Aldrich, N. J., \& Tenenbaum, H. R. (2011). Does discovery-based instruction enhance learning? Journal of Educational Psychology, 103(1), 1-18. https://doi.org/10.1037/a0021017.

Assor, A., Kaplan, H., \& Roth, G. (2002). Choice is good, but relevance is excellent: autonomy-enhancing and suppressing teacher behaviours predicting students' engagement in schoolwork. British Journal of Educational Psychology, 72(2), 261-278. https://doi.org/10.1348/000709902158883.

Bildungsdirektion Kanton Zürich. (2017). Zürcher Lehrplan 21. Zürich: Bildungsdirektion des Kantons Zürich.

Bohl, T., \& Kucharz, D. (2010). Offener Unterricht heute. Konzeptionelle und didaktische Weiterentwicklung. Weinheim: Beltz. 
Bohl, T., Batzel, A., \& Richey, P. (2011). Öffnung - Differenzierung - Individualisierung - Adaptivität. Charakteristika, didaktische Implikationen und Forschungsbefunde verwandter Unterrichtskonzepte zum Umgang mit Heterogenität. Schulpädagogik heute, 2(4), 1-23.

Bos, W., Strietholt, R., Goy, M., Stubbe, T. C., Tarelli, I., \& Hornberg, S. (Hrsg.). (2010). IGLU $2006-$ Dokumentation der Erhebungsinstrumente. Münster: Waxmann.

Bray, B., \& McClaskey, K. (2015). Make learning personal. The what, who, wow, where, and why. Thousand Oaks: Corwin.

Byrne, B. M. (2012). Structural equation modeling with Mplus. New York: Routledge.

Deci, E., \& Ryan, R. (1993). Die Selbstbestimmungstheorie der Motivation und ihre Bedeutung für die Pädagogik. Zeitschrift für Pädagogik, 39(2), 223-238.

DfES (2004a). A national conversation about personalised learning. London. http://webarchive. nationalarchives.gov.uk/20130401151715/http://www.education.gov.uk/publications/ eOrderingDownload/DfES\%200919\%20200MIG186.pdf. Zugegriffen: 6. Juni 2020.

DfES (2004b). Department for education and skills: five year strategy for children and learners. Norwich. http://www.educationengland.org.uk/documents/pdfs/2004-five-year-strategy.pdf. Zugegriffen: 6. Juni 2020.

Dumont, H. (2019). Neuer Schlauch für alten Wein? Eine konzeptuelle Betrachtung von individueller Förderung im Unterricht. Zeitschrift für Erziehungswissenschaft, 22(2), 249-277. https://doi.org/10. 1007/s11618-018-0840-0.

Evans, M., \& Boucher, A.R. (2015). Optimizing the power of choice: Supporting student autonomy to foster motivation and engagement in learning. Mind, Brain, and Education, 9(2), 87-91. https://doi. org/10.1111/mbe.12073.

Furtak, E. M., \& Kunter, M. (2012). Effects of autonomy-supportive teaching on student learning and motivation. The Journal of Experimental Education, 80(3), 284-316. https://doi.org/10.1080/00220973. 2011.573019.

Gärtner, H. (2010). Wie Schülerinnen und Schüler ihre Lernumwelt wahrnehmen. Zeitschrift für Pädagogische Psychologie, 24(2), 111-122. https://doi.org/10.1024/1010-0652/a000009.

Götz, T., \& Nett, U.E. (2017). Selbstreguliertes Lernen. In T. Götz (Hrsg.), Emotion, Motivation und selbstreguliertes Lernen (S. 143-184). Paderborn: Schöningh.

Hagenauer, G. (2011). Lernfreude in der Schule. Münster: Waxmann.

Hartinger, A. (2005). Verschiedene Formen der Öffnung von Unterricht und ihre Auswirkung auf das Selbstbestimmungsempfinden von Grundschulkindern. Zeitschrift für Pädagogik, 51(3), 397-414.

Hartinger, A. (2006). Interesse durch Öffnung des Unterrichts - wodurch? Unterrichtswissenschaft, 34(3), 272-288.

Hartley, D. (2008). Education, markets and the pedagogy of personalisation. British Journal of Educational Studies, 56(4), 365-381.

Hattie, J. (2013). Lernen sichtbar machen. Baltmannsweiler: Schneider-Verlag Hohengehren.

Helm, C. (2014). Erfassung von Freiheitsgraden im Rechnungswesenunterricht mittels Latent Class Analysis. In I. Benischek, A. Forstner-Ebhart, H. Schaupp \& H. Schwetz (Hrsg.), Empirische Forschung zu schulischen Handlungsfeldern (S. 311-335). Wien: Facultas.

Helm, C. (2016). Lernen in offenen und traditionellen UnterrichtsSettings (LOTUS): Empirische Analysen zur Kompetenzentwicklung im Fach Rechnungswesen sowie zum kooperativen, offenen Lernen. Münster: Waxmann.

Helmke, A. (2009). Unterrichtsqualität und Lehrerprofessionalität. Diagnose, Evaluation und Verbesserung des Unterrichts. Seelze-Velber: Kallmeyer.

Holmes, W., Anastopoulou, S., Schaumburg, H., \& Mavrikis, M. (2018). Personalisiertes Lernen mit digitalen Medien. Ein roter Faden. Stuttgart: Robert Bosch Stiftung.

Hoz, V. G. (1986). Educación Personalizada. Madrid: Rialp.

Hu, L., \& Bentler, P. M. (1999). Cutoff criteria for fit indexes in covariance structure analysis: conventional criteria versus new alternatives. Structural Equation Modeling: A Multidisciplinary Journal, 6(1), 1-55. https://doi.org/10.1080/10705519909540118.

IBM Corp (2020). IBM SPSS Statistics for Macintosh, version 27.0. Armonk: IBM Corp.

Jang, H., Reeve, J., \& Deci, E.L. (2010). Engaging students in learning activities: It is not autonomy support or structure but autonomy support and structure. Journal of Educational Psychology, 102(3), 588-600. https://doi.org/10.1037/a0019682.

De Jong, R., \& Westerhof, K.J. (2001). The quality of student ratings of teacher behaviour. Learning Environments Research, 4(1), 51-85.

Katz, I., \& Assor, A. (2007). When choice motivates and when it does not. Educational Psychology Review, 19(4), 429-442. https://doi.org/10.1007/s10648-006-9027-y. 
Keamy, K., Nicholas, H., Mahar, S., \& Herrick, C. (2007). Personalising education: from research to policy and practice. Melbourne: Department of Education and Early Childhood Development.

Kleinke, K., Schlüter, E., \& Christ, O. (2017). Strukturgleichungsmodelle mit Mplus. Berlin: De Gruyter.

Klieme, E., \& Warwas, J. (2011). Konzepte der Individuellen Förderung. Zeitschrift für Pädagogik, 57(6), 805-818.

Lebreton, J. M., \& Senter, J.L. (2008). Answers to 20 questions about interrater reliability and interrater agreement. Organizational Research Methods, 11(4), 815-852.

Lee, D., Huh, Y., Lin, C. Y., \& Reigeluth, C.M. (2018). Technology functions for personalized learning in learner-centered schools. Educational Technology Research and Development. https://doi.org/10. 1007/s11423-018-9615-9.

Lipowsky, F. (1999). Lernzeit und Konzentration. Grundschulkinder in offenen Lernsituationen. Die Deutsche Schule, 91(2), 232-245.

Lipowsky, F. (2002). Zur Qualität offener Lernsituationen im Spiegel empirischer Forschung - Auf die Mikroebene kommt es an. In U. Drews \& W. Wallrabenstein (Hrsg.), Freiarbeit in der Grundschule: Offener Unterricht in Theorie, Forschung und Praxis (S. 126-159). Frankfurt a.M.: Arbeitskreis Grundschule.

Lipowsky, F. (2009). Unterricht. In E. Wild \& J. Möller (Hrsg.), Pädagogische Psychologie (S. 73-101). Heidelberg: Springer.

Lipowsky, F., \& Lotz, M. (2015). Ist Individualisierung der Königsweg zum erfolgreichen Lernen? Eine Auseinandersetzung mit Theorien, Konzepten und empirischen Befunden. In G. Mehlhorn, F. Schulz \& K. Schöppe (Hrsg.), Begabungen entwickeln \& Kreativität fördern (S. 155-219). München: kopaed.

Little, T. D., Cunningham, W. A., Shahar, G., \& Widaman, K. F. (2002). To parcel or not to parcel: exploring the question, weighing the merits. Structural Equation Modeling: A Multidisciplinary Journal, 9(2), 151-173. https://doi.org/10.1207/S15328007SEM0902_1.

Little, T.D., Rhemtulla, M., Gibson, K., \& Schoemann, A. M. (2013). Why the items versus parcels controversy needn't be one. Psychological Methods, 18(3), 285-300. https://doi.org/10.1037/a0033266.

Lüdtke, O., Robitzsch, A., Trautwein, U., \& Kunter, M. (2009). Assessing the impact of learning environments: how to use student ratings of classroom or school characteristics in multilevel modeling. Contemporary Educational Psychology, 34(2), 120-131. https://doi.org/10.1016/j.cedpsych.2008.12. 001.

Marsh, H.W., Lüdtke, O., Nagengast, B., Morin, A.J.S., \& von Davier, M. (2013). Why item parcels are (almost) never appropriate: two wrongs do not make a right-camouflaging misspecification with item parcels in CFA models. Psychological Methods, 18(3), 257-284. https://doi.org/10.1037/ a0032773.

Miliband, D. (2006). Choice and voice in personalised learning. In OECD (Hrsg.), Pesonalising Education (S. 21-30). Paris: OECD.

Müller, A. (2014). Schule kann auch anders sein - Personalisiertes Lernen: das Modell Beatenberg. Eine hintergründige Praxisbeschreibung mit vielen Beispielen und Materialien. Bern: hep.

Müller-Kuhn, D., Häbig, J., Zala-Mezö, E., Strauss, N.-C., \& Herzig, P. (2020). „So richtig Einfluss auf den Unterricht haben wir nicht“ - Wie Schülerinnen und Schüler Partizipation wahrnehmen. In S. Gerhartz-Reiter \& C. Reisenauer (Hrsg.), Partizipation und Schule (S. 187-206). Berlin Heidelberg: Springer.

Murphy, M., Redding, S., \& Twyman, J.S. (2016). Handbook on personalized learning for states, districts, and schools. Philadelphia: Center on Innovations in Learning, Temple University.

Muthén, L. K., \& Muthén, B. O. (2017). Mplus user's guide (8. Aufl.). Los Angeles: Muthén \& Muthén.

OECD (2006). Personalising education. Paris: OECD.

OECD Voice and choice. Schooling for Tomorrow: Knowledge bank. https://www.oecd.org/site/ schoolingfortomorrowknowledgebase/themes/demand/voiceandchoice.htm. Zugegriffen: 6. Juni 2020.

Oelkers, J. (2010). Reformpädagogik: Entstehungsgeschichten einer internationalen Bewegung. Zug: Klett.

Pauli, C., Reusser, K., \& Grob, U. (2007). Teaching for understanding and/or self-regulated learning? A video-based analysis of reform-oriented mathematics instruction in Switzerland. International Journal of Educational Research, 46(5), 294-305.

Pauli, C., Reusser, K., \& Grob, U. (2010). Reformorientierter Mathematikunterricht in der Deutschschweiz. In K. Reusser, C. Pauli \& M. Waldis (Hrsg.), Unterrichtsgestaltung und Unterrichtsqualität. Ergebnisse einer internationalen und schweizerischen Videostudie zum Mathematikunterricht (S. 309-339). Münster: Waxmann. 
Pauli, C., Reusser, K., Waldis, M., \& Grob, U. (2003). „Erweiterte Lehr- und Lernformen“ im Mathematikunterricht der Deutschschweiz. Unterrichtswissenschaft, 31(4), 291-320.

Prain, V., Cox, P., Deed, C., Dorman, J., Edwards, D., Farrelly, C., et al. (2013). Personalised learning: lessons to be learnt. British Educational Research Journal, 39(4), 654-676.

Rakoczy, K. (2006). Motivationsunterstützung im Mathematikunterricht. Zur Bedeutung von Unterrichtsmerkmalen für die Wahrnehmung von Schülerinnen und Schüler. Zeitschrift für Pädagogik, 52(6), 822-843.

Rakoczy, K., Buff, A., \& Lipowsky, F. (2005). Befragungsinstrumente. In E. Klieme, C. Pauli \& K. Reusser (Hrsg.), Dokumentation der Erhebungs- und Auswertungsinstrumente zur schweizerisch-deutschen Videostudie „Unterrichtsqualität, Lernverhalten und mathematisches Verständnis“. Materialien zur Bildungsforschung, Bd. 13. Frankfurt am Main: GFPF.

Ramm, G., Prenzel, M., Baumert, J., Blum, W., Lehmann, R., Leutner, D., Neubrand, M., Pekrun, R., Rolff, H. G., Rost, J., Schiefele, U., \& PISA-Konsortium Deutschland (2006). PISA 2003: Dokumentation der Erhebungsinstrumente. Münster: Waxmann.

Reusser, K. (2011). Von der Unterrichtsforschung zur Unterrichtsentwicklung - Probleme, Strategien, Werkzeuge. In W. Einsiedler (Hrsg.), Unterrichtsentwicklung und didaktische Entwicklungsforschung (S. 11-40). Bad Heilbrunn: Klinkhardt.

Reusser, K., \& Pauli, C. (2010). Unterrichtsgestaltung und Unterrichtsqualität - Ergebnisse einer internationalen und schweizerischen Videostudie zum Mathematikunterricht: Einleitung und Überblick. In K. Reusser, C. Pauli \& M. Waldis (Hrsg.), Unterrichtsgestaltung und Unterrichtsqualität (S. 9-32). Münster: Waxmann.

Reusser, K., Stebler, R., Mandel, D., \& Eckstein, B. (2013). Erfolgreicher Unterricht in heterogenen Lerngruppen auf der Volksschulstufe des Kantons Zürich. Zürich. http://edudoc.ch/record/108236/files/ Vielfalt_Volksschule_Bericht.pdf. Zugegriffen: 6. Juni 2020.

Rioux, C., Stickley, Z.L., Odejimi, O. A., \& Little, T. D. (2020). Item parcels as indicators: why, when, and how to use them in small sample research. In R. van de Schoot \& M. Miocević (Hrsg.), Small sample size solutions: a guide for applied researchers and practitioners (S. 203-214). London, New York: Taylor \& Francis.

Schratz, M., \& Westfall-Greiter, T. (2010). Das Dilemma der Individualisierungsdidaktik. Plädoyer für personalisiertes Lernen in der Schule. Journal für Schulentwicklung, 1, 18-31.

Sebba, J., Brown, N., Steward, S., Galton, M., \& James, M. (2007). An investigation of personalised learning approaches used by schools. London: Department for Education and Skills.

Stebler, R., Pauli, C., \& Reusser, K. (2018). Personalisiertes Lernen. Zur Analyse eines Bildungsschlagwortes und erste Ergebnisse aus der perLen-Studie. Zeitschrift für Pädagogik, 64(2), 159-178.

Stebler, R., Pauli, C., \& Reusser, K. (2021). Personalisiertes Lernen als schulisches Bildungskonzept. Erscheinungsformen, Qualitätsmerkmale und Forschungsbefunde. In G. Brägger \& H.-G. Rolff (Hrsg.), Handbuch Lernen mit digitalen Medien (S. 402-430). Weinheim: Beltz.

U.S. Department of Education (2017a). Reimagining the role of technology in education: 2017 national education technology plan update. Washington: Office of Educational Technology.

U.S. Department of Education (2017b). What is personalized learning? Office of educational technology. https://medium.com/personalizing-the-learning-experience-insights/what-is-personalized-learningbc874799b6f. Zugegriffen: 6. Juni 2020.

Wagner, W. (2008). Methodenprobleme bei der Analyse der Unterrichtswahrnehmung aus Schülersichtam Beispiel der Studie DESI (Deutsch Englisch Schülerleistungen International) der Kultusministerkonferenz. Dissertation. Koblenz-Landau: Universität Koblenz-Landau. 